\title{
NEXT GENERATION NETWORKS - A VISION OF NETWORK EVOLUTION
}

\author{
Howard Green ${ }^{1}$, Pierpaolo Ghiggino ${ }^{1}$ \\ ${ }^{I}$ Marconi Communications, Stoneleigh House, New Century Park, Coventry, CV3 1HJ, UK
}

\begin{abstract}
This article presents a view of the needs and developments for the "Next Generation Networks". It starts from a market and service context following the burst of the Internet bubble and sketches the likely evolution of services by end user type. It is centered, however on a vision of network evolving to architectures necessary to support the needs of operators with special emphasis on the European environment.
\end{abstract}

\section{MARKET \& SERVICE CONTEXT}

\subsection{Network traffic}

Total service revenues are still increasing, but revenue segmentation in service categories shows a move towards broadband access and mobile (see Fig. 1,2)
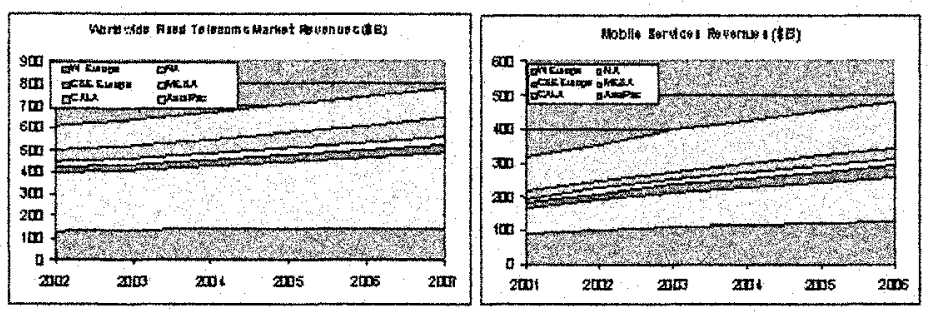

Figure $1 \& 2$ - Global fixed and mobile revenues by region (Source Gartner) 
The dominating revenue stream remains voice traffic. On a worldwide basis, fixed network voice revenues are largely flat. Growth is mostly in new markets, offset by decline in mature areas (most pronounced in Western Europe). Prices continue to fall where competition exists. Mobile call volumes and revenues are continuing to grow quite rapidly (although prices are falling, and there is some evidence that revenue growth is slowing slightly). Mobile telephony revenues are set to exceed fixed line revenues in the near future ${ }^{1}$. In many less developed markets, mobile telephony is supplying the basic voice communications need, as fixed line infrastructure is not available.

In general, fixed line network operators in the developed world are expecting a slow long-term decline in revenues from these traditional services. They are developing defensive strategies (pricing tactics against mobile services, for example) but nonetheless expect these revenues to decline (3\% per annum is a European operator expectation).

\subsection{Service evolution}

\subsubsection{Residential Customers}

Residential broadband is a major success as a consumer technology. Worldwide, the dominant method of provision is DSL. In the UK and US, cable modem has also been a major success (see fig. 3 for the UK scenario).

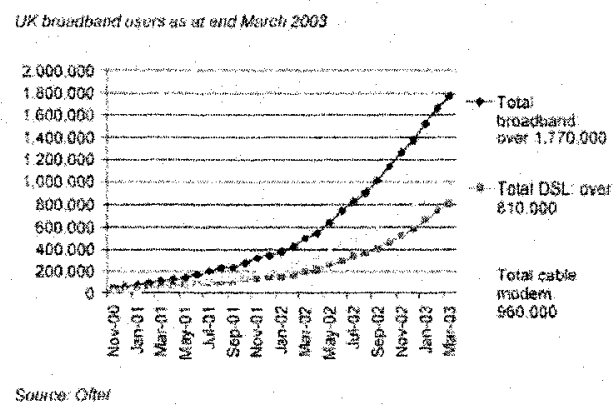

Fig. 3 - UK Broadband end user base growth

${ }^{1}$ A major source of recent fixed line revenue has been calls to mobile networks. These prices are now being reduced by regulators. 
It is expected that residential broadband services will be a key growth area over the next 5 years or so, growing at a faster rate than business services, to become one of the most important revenue segments.

Broadband service users typically consume 10 times the bandwidth of narrowband users per person, while revenues are simply based on subscription (no more than twice a typical narrowband subscription). This causes a decrease in revenue per bit carried, leading to cost pressure within service provider networks.

Increase in revenue is likely to be generated not just by higher penetration of broadband for fast Internet access, but also by additional services. For this to be profitable, however, such new services need to be made available without significant additional infrastructure investment.

Such services are likely to be based on specific content and application needs, such as (personal interest) video-on-demand and online gaming via specialized game consoles (e.g. PS-2). Whilst the business models have yet to evolve, there are two likely scenarios. Firstly, the ISP could become the focus (via their portal) from which end-users purchase such content and applications direct [the ISP as "media aggregator"]. Secondly, such purchases could be made from the content / application owners, with a proportion of the revenue going to the service provider for such things as portal placement, localized caching and (later) session-based services providing increased capacity and/or QoS. (The two models can be thought of as a retail scenario and a wholesale scenario.)

Within a few years, typical residential customers will use some sort of home media server. Via in-home networking technologies (typically 802.11 WLAN), these will connect using xDSL transport to such services in an "always-on" manner.

An increasing amount of voice traffic will be handled by mobile networks. What remains on the fixed networks could be decreased further (in PSTN terms) by VoIP, because of voice-enabled game software, instant messaging packages $\&$ multimedia contact centres.

An important opportunity for the fixed network operator is to capture parts of the mobile revenue stream by facilitating fixed/mobile convergence. For example:

- Services allowing terminal mobility from the mobile to the fixed network e.g. use of a WiFi enabled mobile terminal on fixed network tariffs at home using packet voice over DSL.

- Integration of fixed and mobile directory services, and the use of "presence" applications to keep in touch with people.

An increasing percentage of people are working from home, at least on a parttime basis. Secure IP VPNs through the Internet will become the predominant way to connect such teleworkers to their corporate networks. 


\subsubsection{Small and Medium Enterprises (SMEs)}

Similar to the situation with residential broadband, business broadband over DSL is expected to show healthy year-by-year growth.

As DSL products were initially developed for residential customers and priced for the mass market, these services can prove very attractive to SMEs. On the downside, there is a significant risk of substitution ("cannibalization") of existing leased line revenues.

Service performance and guaranteed SLAs are the main means of differentiation with leased line services, as customers are willing to pay a premium if their specific needs are met with distinct products. However, the delivery mechanism is not as important to SMEs as the service provided for a given cost.

It is likely then that data VPN services will grow rapidly where they are offered over xDSL broadband access. Such provision could then result in service providers offering seamless service evolution from xDSL technologies towards higher capacity fiber based solutions.

It can be expected that, where SMEs operate over multiple sites, service provider provision of data VPN services could become much more attractive than the renting of leased lines and self-provisioning of services on them.

Coupled to this, customers will expect to get a multitude of services delivered at a single interface (with appropriate quality. This requires technical mechanisms for service separation (e.g. VLAN tags, MPLS labels, ATM VCs), session admission control and flow policing so as to provide appropriate guarantees of quality and security.

VoIP/Centrex will be among the interesting business options for such customers based on advanced broadband access.

\subsubsection{Large Corporate Customers}

Large corporate customers are examining their WAN costs as part of reviews of IT infrastructure, and will expect to continue to get more bandwidth and lower prices. Nonetheless, there are opportunities to increase overall communications revenue, in particular by convincingly demonstrating that other costs can be avoided using communications. Such opportunities are likely to arise in respect of supply chain integration, outsourcing, travel avoidance, and reduction in fixed office costs. There is also an increased focus on network security and disaster recovery, leading to requirements for network-wide encryption and authentication, and to the need for geographically remote storage backup.

Centralized servers organized in hub-and-spoke configuration are a common model. The dominating technologies used to build such WANs are FR and ATM, 
as well as SDH-based leased line networks, but growth in data VPN services is expected to eat into this.

There is a spectrum of these data VPN services, ranging from "multiplexed Ethernet private line" (multiple "Ethernet virtual circuits" from a large site switched to different destinations in the network on the basis of VLAN tag), through "complex L2" VPN technologies (such as Virtual Private LAN Service (VPLS) - formerly "draft Lassere-Vkompella") to L3 VPN technologies based on IP/MPLS routing (e.g. RFC 2547) to VPNs based on IPSec. From the service perspective, these offerings can be differentiated by scalability, security and QOS characteristics (where large numbers of endpoints are involved, L3 routing is necessary, and delay-sensitive intersite services still require leased line-like QOS guarantees). Not all of these services will find favour in the marketplace. Moreover, many operators are finding these services more complex to provide and administer than traditional leased lines, whilst service prices are usually lower.

Home worker and Telecommuters now frequently use the Internet to access company servers by means of secure tunneling. Utilization of wireless LANs is currently quite low, but likely to expand within both the corporate environment, and via the use of Wireless hot spots.

ISDN PBX is the predominant infrastructure for corporate voice. There is no compelling short-term reason to replace this infrastructure, however as replacement opportunities arise, packet voice will be the new technology of choice. VoIP PBXs are already taking substantial market share. An increasing amount of these packet voice service opportunities will be outsourced to service providers. There will also be business development of PC instant messaging products to provide "virtual presence" and interface via VoIP with mobile networks.

The physical service interface is likely to be fast or Gigabit Ethernet, allowing for flow separation by VLAN tags or MPLS labels, and providing a "fractional rate" service ${ }^{2}$.

\section{IMPACT ON OPERATORS}

After the "bursting of the Internet bubble", network operators are under significant business pressure. As consequences, operators have two major objectives:

1. Need for substantial reduction of ongoing network operational costs, and incremental capacity at much lower cost per bit.

2. Need for new service revenues. There is unlikely to be any single "killer application", and so this likely requires many new services to be deployed.

2 That is, a "subscribed bit rate" less than physical line rate, policed by the network, and variable in relatively small increments by service provisioning. 
Operators are at different stages of emergence from corporate reconstruction and debt reduction programs. Moreover, they have different views of the appropriateness of their basic network infrastructure. Nonetheless, they are increasingly beginning to consider new programs of capital investment to address the objectives above (OPEX reduction and multi-service flexibility). These investment programs will have major consequences both in network and in OSS architecture for operators.

\section{NETWORK CONSEQUENCES}

Operators are discovering a real business need to make convergence happen. Reducing the number of edge platforms deployed in high volume in their networks, and reducing the number of service-specific overlay networks are major opportunities for increased efficiency and cost reduction. They need to find new service revenues from added value on top of basic broadband and new services to small business. These new services need to be delivered with appropriate quality, and will need usage-based charging.

\subsection{Operational Support Systems (OSS) consequences}

In mature organizations there are opportunities to rationalize operational support systems (which are often based on individual service needs rather than common business processes). The rationalizations may be linked with network evolution but can also take place independently. For example, a flexible billing system operating across many services and several networks can be introduced independently of the evolution of the networks. A common fault management process can be introduced in a similar way. There are also opportunities to introduce new processes and systems where there may be nothing today: for example post processing of usage records can help to improve fraud detection and reduce revenue 'leaks'. Introduction of auto-discovery algorithms and centralized inventory may assist in validating network equipment usage and result in better utilization of capacity. Where there are demonstrable short to medium term returns on investment operators will invest in OSS in order to:

- Improve operational efficiency - minimize data entry, end user control, error reduction, supply chain integration, fast fulfillment, speed of fault location and repair.

- Improve capital investment utilization - accurate inventory, efficient utilization of installed capacity, timely investment in new capacity

- Increase/secure revenue - flexibility of service definition, bundling and tariffing, improved customer retention, revenue assurance 


\section{A NETWORK VISION}

Future communication networks will provide a significantly broader range of services. As profit margins will continue to be low, these networks will need to be realized by a minimum number of technologies ${ }^{3}$.

IP will be the near-universal technology of the service layer. MPLS will eventually form a common virtual-circuit switching backbone layer, running over an SDH/OTN transport core (with integrated DWDM). Specialized high capacity traffic streams (e.g. SAN) will be mapped with minimum overhead onto core transport. For high capacity business services, the access and metro networks will have a traffic aggregation and transport role, connecting customer IP routers to IP service switches at the "edge of the core". For residential broadband and SMEs, a multi-service access node will manage the continuing diversity of "last mile" technologies (including copper, wireless and some fibre). (See Fig. 4 \& 5)
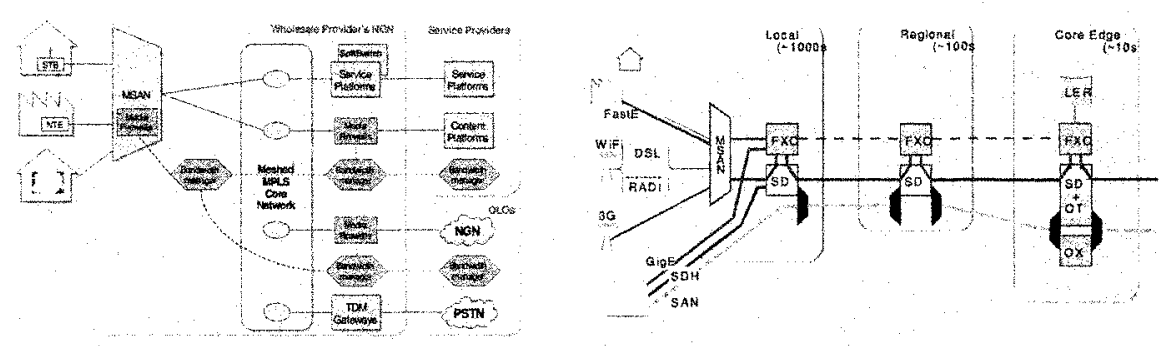

Fig. $4 \& 5-$ Next Generation Network structure and transport views

\subsection{Customer premises Equipment}

As the network simplifies, most of the required network terminals will become standardized consumer products. Currently we already have standardized ADSL and cable modems, and integration with "Internet gateway" switches and firewalls. We believe that this is likely to extend into the business market. Of particular significance is the Ethernet in the First Mile (EFM) initiative, which may well create a market for both copper and fibre termination of fractional Ethernet (and possibly also E1 \& Ethernet together over fibre). There will remain a market for low cost Ethernet over SDH terminals, and for access C/DWDM.

${ }^{3}$ For a broader description of some key aspects of such technologies see $\mathrm{H}$ Green, $\mathrm{P}$ Ghiggino: "An overview of key technologies for the next generation networks" Submitted to OpNeTec 2004 


\subsection{Edge Transport}

Access and metro transport will be based on Ethernet L2 aggregation techniques, using frame labels such as VLAN tags or MPLS for "permanent virtual circuits" with fine bandwidth granularity. Both in the access and regional transport area, these frame streams may be carried either as native Ethernet (with OAM additions such as those proposed by EFM) or GFP encoded to SDH virtual concatenation groups. SDH will continue to be used for high-quality synchronous transport (E1s for PBX interconnect, mobile backhaul etc.) especially where timing/clock distribution requirements are important, and is a natural choice where ring topology protection is useful.

While transport devices will evolve the capability to cross-connect labeled frame streams, we do not believe that this will imply the need for a full IP/MPLS control plane. There will be a move towards more automatic provisioning of these label switched paths, most likely based on ASTN concepts. A vital feature of this architecture is that these aggregation nodes should be simple to configure and maintain, and should be "transparent" to the service level interaction. For this reason, we believe that such devices should offer only a limited range of traffic management/statistical multiplexing options (essentially committed effort and best effort concentration) which can be policed once at ingress to the network.

\subsection{Multi-Service Access}

Multi-service access platforms, located at copper termination points (and perhaps also in street cabinets) are a vital part of the next generation network. They will support a graceful evolution from POTS to packet voice (using "combo" line cards and integrated packet voice gateways), and allow a range of value-added services to be deployed on top of residential broadband access. They will gradually replace many different service-specific devices at the edge of the network, allowing significant simplification and operational cost saving, whilst allowing continued diversity of "last mile" options.

MSANs can be seen as a new generation of DSLAMs. As such, they need to maintain the ATM aggregation capabilities of DSLAMs on the line side. In our view, ATM remains an effective way of managing controlled multiplexing of services on relatively low-speed lines. On the aggregate side, we expect MSANs to offer Ethernet transport (native and NG-SDH).

Current generation DSLAMs transport ATM VCs per customer back into the network, where they are terminated at a B-RAS. The B-RAS forms the point of service termination, calling upon external servers (e.g. RADIUS) for the so-called "triple-A" (Authorization, Authentication, \& Accounting) functions, and then forward the virtual circuits to ISP edge routers using IP Layer 2 Tunneling protocol 
(L2TP). This architecture does not scale well, especially when added value services require multiple classes of service per customer. Hence we believe that this B-RAS functionality will be distributed to the MSANs, which will terminate multiple ATM VCs per customer, and directly map to L2TP inside provisioned MPLS tunnels for different QOS classes (or use "draft Martini" MPLS label stacking). The MSAN will continue to use centralized servers for "triple-A"4.

\subsection{MPLS Core}

In current large data networks, there are often three kinds of network elements: edge routers, core routers, and L2 switches (usually ATM).

These architectures are rightly felt to be complicated to configure and difficult to maintain. The router elements have no concept of differentiated service quality, and hence there is required to be an underlying network of virtual circuits separating traffic.

Because of resilience and control plane scaling issues, routers are often arranged in complex hierarchies, implying many packet hops and long latency.

For these reasons most operators believe that their current generation core packet networks will require major change to make them suitable as the core of a large multi-service network offering service guarantees. We believe that there will be two kinds of network element remaining:

1. Large $(\sim 1 \mathrm{~Tb} / \mathrm{s})$ "edge of core" MPLS switch/routers, arranged in a "sparse mesh" ${ }^{\text {" }}$ topology, forming a universal network core.

2. IP service switches for high-value services (e.g. IPSec VPN) only accessed as needed.

[This assumes a "wholesale" model of services, where the core network provider is connecting customers to multiple service providers, whose switches are accessed through the core. Where the core network operator is providing retail services directly (e.g. RFC 2547 VPNs, internet access) there may be a need for IP services nodes "before the core"]

MPLS core switches will also need to interwork with remaining ATM-based network services (which, like TDM, will have a long "old age").

${ }^{4}$ Whilst this does imply some distribution of complexity, it remains service agnostic. Thus new services can be added without impact on high-volume network elements

${ }^{5}$ That is, high-usage routes are all directly connected. Some lower-usage routes may utilise transit nodes. Where required simply by geography, transit is probably better provided at the transport layer. 


\subsection{Core transport}

It has been suggested that a next-generation network does not need a core transport layer, since it can be formed simply from "IP over light" - that is, IP routers connected together using high capacity optical (perhaps DWDM) interfaces. We think by contrast that there continues to be an important role for a core transport network at VC4/ODU granularities, for the following reasons:

1. There will still be a significant quantity of "leased line" business inter-site services. Even if these are presented as "Ethernet Private Line", there is no need to use expensive router capacity to provide these services. Crossconnection in the transport layer is a lower-cost option and easier to administer (especially when using ASTN-based automated provisioning, in which explicitly routed paths are downloaded from the network planning process).

2. The mesh core topology envisaged above requires the interconnection of large numbers of MPLS nodes at bit rates in the range $\sim 100 \mathrm{Mb} / \mathrm{s}$ to $20 \mathrm{~Gb} / \mathrm{s}$. This requires a "virtual port" technology allowing single high capacity physical interfaces to be routed to different destinations. The most plausible options are STM-64/256 canalized at STM1, and GigE/10GigE using MPLS or VLAN tags. In either case, there is need of a transport layer to "fan out" these interfaces, and to arrange for transit where required (in the Ethernet case, the transport network must map tags to VC4 or ODU1 granularity VCGs).

3. Large networks of routers take a considerable time to respond to faults. Transport-based protection can handle most common faults in $<50 \mathrm{msec}$ timescales, avoiding any impact on the router layer.

This transport layer will be built from large SDH/OTN cross-connects with integrated WDM and a sophisticated ASTN control plane.

\subsection{Control Plane Scalability}

Many of the key challenges to next generation network design lie in this area. As we have argued, the network will require underlying resource reservation mechanisms to ensure appropriate quality in supplying a wide range of services. This requires a concept of "virtual circuit". VCs are likely to be implemented in the network using several base technologies (at least MPLS, ATM \& Ethernet VLAN tagging) which will need to interwork.

It is very easy to design network architectures involving meshes of virtual circuits which require impossibly large numbers of circuits, or "automatic discovery" architectures sharing vast amounts of information. In general, our view is that the network continues to need to be partitioned both horizontally (hierarchical layers) and vertically (access/core), but that complexity can be reduced by using similar mechanisms in each domain. Individual service types will 
in general use "virtual networks" consisting of provisioned tunnels. Thus, for example, most services will need to use IP routing (Label Edge Router functionality) as they enter the network core. To give an example, Internet access packets destined for a single ISP will be unpacked from access LSPs (at least 1 per MSAN) and repacked to a single LSP.

\subsection{Bandwidth management}

For a multi-service network providing service guarantees, it is not enough just to reserve resources for appropriate traffic aggregates. It is also necessary to operate admission control for services requiring the guarantees, so as to ensure that the aggregates are not over-filled. This issue is already recognized for packet voice, but in a multi-service network is applicable to all guaranteed services. Admission control requires a count of resource used throughout the network, to decide whether a new service instance can be admitted.

This counting of resource for admission control can in principle be done at least three ways.

1. Directly by reserving the resource by signalling through the set of network nodes (using RSVP, for example)

2. In the application, for example using circuit and route control in a softswitch

3. Using a separate logical layer of "bandwidth managers"

We believe it is important that next generation networks allows common mechanisms to support these functions for all services needing guarantees, and thus we think this function should be separated from individual applications. For this reason we generally support the creation of a "bandwidth management" function with a standardized application interface. The bandwidth management layer will also need to communicate across operator boundaries, and hence standardization is essential. There is work going on (for example, in ETSI Tiphon and the MultiSwitching Forum) on architectures and protocols for such bandwidth managers.

\subsection{Service Platforms and Gateways}

The network described above is capable of carrying the full range of services. Of course such a network will still need a variety of platforms to support the logic of particular service types, and to perform specialized data plane functions (e.g. hardware support for IPSec encryption). Our view is that these platforms will be subtended as "server pools" from the network core. This allows them to be used only as required, and gives great flexibility for centralization of resource, networkwide redundancy etc.

Similarly gateways to the TDM network and to other operators are accessed through the core and can be placed wherever convenient, supporting a view in 
which operators can use building space more flexibly and concentrate resource at fewer locations.

\section{CONCLUSION}

We have presented a view of the development of next generation networks. It is being driven by:

- Flat to declining revenues from traditional fixed line services

- Rapid growth in bandwidth demand, predominantly from data services

- Reducing revenues per bit carried

- Business need for major operating cost reduction

A set of emerging technologies does allow operators to address these business problems.

- Emergence of Internet Protocol as an increasingly ubiquitous service interface

- A wide variety of broadband access technologies (xDSL, optical Ethernet, Wireless) supporting a common IP service framework

- Data-enabled transport, including "Next Generation SDH" and Ethernet developing for the WAN, utilising a control plane for fast restoration and implementation of provisioning.

- Maturing MPLS standards and implementations supporting packet Quality of Service

The resulting network architecture is likely to be characterised by:

- Multi-service access - a single family of platforms supporting the full range of services at the network edge, with traditional access and transport coming together

- Aggregation of packet flows in data-aware transport devices

- A densely interconnected network core of large MPLS nodes, supported by intelligent transport providing resilience, flexibility and fan-out.

Service nodes and network gateways located as "server pools" at convenient locations accessed through the network core. 